\title{
Frequency-dependent effects of oscillatory-tDCS on EEG oscillations: a study with Better OSCillation detection method (BOSC)
}

\author{
A. D'ATRI' ${ }^{12}$, E. DE SIMONI', M. GORGONI', M. FERRARA³, F. FERLAZZO', \\ P.M. ROSSINI ${ }^{2,4}$, L. DE GENNARO ${ }^{1,2}$ \\ ' Department of Psychology, University of Rome "Sapienza", Rome, Italy; ${ }^{2}$ IRCCS San Raffaele Pisana, \\ Rome, Italy; ${ }^{3}$ Department of Life, Health and Environmental Sciences, University of L'Aquila, L'Aquila, Italy; \\ ${ }^{4}$ Institute of Neurology, Catholic University of The Sacred Heart, Rome, Italy
}

\begin{abstract}
A B S T R A C T
Oscillating transcranial direct current stimulation (osc-tDCS) modulates the spontaneous brain activity in a frequency-specific manner. Most studies evaluated cortical effects of osc-tDCS through spectral analysis measures, without differentiating components associated with rhythmic and non-rhythmic activity. Since osc-tDCS mainly affects brain oscillatory activity, our aim was to investigate on the specific changes of EEG oscillations following a frontal osc-tDCS at 0.8 and at $5 \mathrm{~Hz}$.

20 healthy subjects $(26.8 \pm 2.5$ years) participated in one of two experiments (Exp. $1=0.8-\mathrm{Hz} t \mathrm{tDCS}, n=10 ;$ Exp. $2=5-\mathrm{Hz}$ tDCS, $n=10$ ), consisting of 3 within-subject sessions: two active conditions with different stimulation polarity (anodal osctDCS, cathodal osc-tDCS), and a control condition (sham).

EEG oscillatory components (28 cortical derivations) at the stimulation frequency were measured by the Better OSCillation detection method (BOSC). Variations between before and after the osc-tDCS were compared between conditions as a function of polarity (anodal vs. cathodal vs. sham) and frequency $(0.8 \mathrm{vs} .5 \mathrm{~Hz})$ of stimulation.

The main finding is a significant local increase of 0.81-Hz slow oscillations $\left(F_{1,18}=19.97 ; p=0.0004\right)$ and 5.3-Hz theta oscillations $\left(F_{1,18}=26.93 ; p=0.0001\right)$ after $5 \mathrm{~Hz}$ compared to $0.8-\mathrm{Hz}$ tDCS.

Our study shows larger frequency-specific and cross-frequency effects of 5- $\mathrm{Hz}$ compared to 0.8-Hz stimulation, not revealed by conventional FFT analyses. This finding is consistent with a more effective induction of EEG synchronization during wakefulness by means of a stimulation in the theta range, and it suggests to combine measurement of EEG power and EEG oscillations in future studies involving transcranial stimulations.
\end{abstract}

\section{Key words}

Transcranial direct current stimulation (tDCS) • Resting EEG • EEG synchronization $\bullet$ EEG oscillations • Frontal cortex $\bullet$ Better OSCillation method (BOSC)

\section{Competing interests}

The authors declare that the research was conducted in the absence of any commercial or financial relationships that could be construed as a potential conflict of interest.

\section{Introduction}

Transcranial current stimulation (tCS) protocols have been frequently applied in both basic neuroscience and clinical fields as tools to non-invasively modulate brain activity. In the last decade, in fact, oscillatory-tDCS (osc-tDCS), in which a polarizing current with a sinusoidal waveform $(0.75 \mathrm{~Hz})$ is applied, has been shown to affect EEG activity at the stimulation frequency 
during NREM and REM sleep (Marshall et al., 2006; 2011) and during active and resting wakefulness (Kirov et al., 2009; Neuling et al., 2012). It has been proposed that the application of a transcranial time-varying current acts mainly on cortical oscillatory activity inducing resonance phenomena between the variations of the exogenous electric field and the spontaneous oscillations of the stimulated cortical networks (Ozen et al., 2010; Fröhlic and McCormick, 2010). In other words, cortical networks are driven by the external field to oscillate with a greater amplitude at the stimulation frequency.

According to this mechanism of action, the application of an anodal osc-tDCS at $0.75 \mathrm{~Hz}$ (slow oscillation-tDCS: so-tDCS) on frontal areas during NREM boosted endogenous slow oscillations and alpha rhythm (Marshall et al., 2006), while a stimulation at $5 \mathrm{~Hz}$ $(\theta$-tDCS), that is within the theta frequency range, resulted in the opposite pattern (Marshall et al., 2011). Moreover, the same anodal $\theta$-tDCS applied during REM sleep appeared to increase gamma activity, suggesting the brain state-dependency of tDCS effects on EEG rhythms (Marshall et al., 2011). When a so-tDCS was applied during wake, it induced a widespread increase of theta activity, while a smaller enhancement of slow oscillations was limited to the cortical sites underneath the electrodes (Kirov et al., 2009). According to the brain state-dependency of the stimulation effects, the increase of theta activity was more pronounced when the stimulation was applied during quiet wakefulness, characterized by an EEG background dominated by alpha and theta rhythms, than during attentive wakefulness, when the EEG background is dominated by higher frequencies. The brain state-dependency of osc-tDCS could be explained by the presence of network's resonance frequencies (Gutfreund et al., 1995; Hutcheon and Yarom, 2000). In physics, a resonance frequency is defined as a frequency at which even a small periodic driving force leads the system to oscillate with a greater amplitude at the given frequency. This notion, when applied to the interaction between stimulation and cortical activity, implies that the closer the stimulation frequency is to the frequency of spontaneous oscillations in the cortical network, the more the stimulation is effective in inducing resonance effects in the network, even at a low current intensity (Radman et al., 2007; Fröhlich and McCormick, 2010; Reato et al., 2010; Ali et al., 2013).

Given these assumptions, we could expect that a stimulation in theta frequency range during wakefulness would entrain cortical oscillations more easily than the so-tDCS applied by Kirov et al. (2009). In fact, the resting state with eyes-closed is characterized by cortical rhythms mainly in alpha and theta frequency band, while slow oscillations (i.e. $<1 \mathrm{~Hz}$ activity) are instead spontaneously prevalent during NREM sleep. In other words, the 5-Hz stimulation frequency being likely closer to the intrinsic oscillations frequency of the target cortical area than the $0.8 \mathrm{~Hz}$, it should result in a more effective action on cortical activity.

According to this hypothesis, the current study investigates for the first time on the effects of a frontal osc-tDCS at $5 \mathrm{~Hz}$ ( $\theta$-tDCS) and at $0.8 \mathrm{~Hz}$ (so-tDCS) during wakefulness with eyes-closed focusing the analysis on cortical oscillations. It should, be considered that conventional spectral-power measures, based on Fast Fourier Transform (FFT) routines, do not clearly differentiate the power associated with rhythmic versus non-rhythmic activity (Caplan et al., 2015). Since oscillating current stimulations increase the synchronization of firing times across neurons (Radman et al., 2007) resulting in more regular oscillations of the stimulated cortical network (Ali et al., 2013),we focused our analysis on changes in "genuine" oscillatory EEG activity as measured by Better OSCillation (BOSC) detection method (Caplan et al., 2001). The Better OSCillation (BOSC) is a method to identifying rhythmic activity at specific frequencies within the background of non-rhythmic EEG components (Caplan et al., 2001; Whitten et al., 2011). The same method has been applied to the EEG recorded in different brain states, disclosing the presence of EEG oscillations even when they are rare or sporadic without a discernible peak in the EEG power spectra (Caplan et al., 2001; Caplan and Glaholt, 2007; Hughes et al., 2012; Marzano et al., 2011, 2013; Moroni et al., 2012; Whitten et al., 2011). In the present study, this analysis allowed to highlight even small and transient changes in oscillatory activity induced by the stimulations.

\section{Methods}

\section{Participants}

Twenty healthy female subjects (18 to 30 years) have been divided in two experimental groups: ten subjects (mean age: $26.2 \pm 2.5$ years) participated in Exp. 1, and ten subjects (mean age: $27.4 \pm 2.4$ 
years) participated in Exp. 2. Exp. 1 and 2 evaluated the after-effects on the resting EEG of an oscillatory tDCS at 0.8 and at $5 \mathrm{~Hz}$, respectively.

All participants gave informed written consent and met the following inclusion criteria: no presence or history of epilepsy, no neurological or psychiatric disorder and intracranial metal implants, no daytime nap habits or excessive daytime sleepiness or other sleep disturbances as assessed by a clinical interview. During the week before the experimental sessions, participants were asked to fill out a daily sleep $\log$ in order to control their keeping constant the wake-sleep cycle. During the morning of experimental sessions they were not allowed to consume coffee, tea, chocolate, or any kind of drugs. All subjects were tested in the luteal phase of the menstrual cycle in order to avoid possible confounding effect of the cyclical ovarian hormones.

The study was approved by the Institutional Ethics Committee of the Department of Psychology of University of Rome Sapienza, and was conducted in accordance with the Declaration of Helsinki.

\section{Experimental design}

The experiments consisted of three within-subject experimental sessions, two active conditions (anodal and cathodal osc-tDCS) and a sham condition, separated by an interval of at least 1 week. The sequence of sessions was partially balanced across subjects.

Regardless of the stimulation features in the different experimental conditions, the timeline of the experimental sessions was identical in both the experiments (Figure 1).

Subjects arrived at the laboratory at 12:00 $\mathrm{h}$ and underwent the preparation for EEG recordings and stimulation. At the end of the electrodes montage, they were asked to sit relaxed on a comfortable chair in a soundproof, temperature-controlled, and electrically shielded room. Each session started at 14:00 $\mathrm{h}$ and included: a) a 5-min EEG recording (prestimulation); b) the stimulation protocol (10 min); c) a 5-min EEG recording (post-stimulation). EEG was recorded in a resting eyes-closed condition. During recordings, subjects were asked to imagine fixating a point on the wall in front of them.

We assessed the stimulation polarity contribution by fixing the stimulation frequency and comparing anodal vs. cathodal oscillatory stimulations, while we investigated the frequency-dependent effects comparing $5 \mathrm{~Hz}$ vs. $0.8 \mathrm{~Hz}$ osc-tDCS at both the stimulation polarities.

\section{EEG recordings}

The EEG signals were recorded from 28 sintered $\mathrm{Ag}-\mathrm{AgCl}$ electrodes mounted in an elastic cap (Easycap, Falk Minow, Munich, Germany) at scalp locations C3, C4, Cp1, Cp2, Cp5, Cp6, Cz, F3, F4, F7, F8, Fc1, Fc2, Fc5, Fc6, Fp1, Fp2, Fz, O1, O2, Oz, P3, P4, P7, P8, Pz, T7, T8 (according to the 10-20 system) with linked mastoid references (A1 and A2). Horizontal eye movements were detected by recording electro-oculograms (EOGs), and the electromyogram (EMG) was recorded by two submental electrodes for off-line artefact detection. The ground electrode was positioned between $\mathrm{Fz}$ and $\mathrm{Cz}$, at Fcz. Electrode resistance was kept below $5 \mathrm{k} \Omega$. Signals were recorded using the BrainAmp MR plus system (Brain Products $\mathrm{GmbH}$, Gilching, Germany) with a sampling rate of $250 \mathrm{~Hz}$, amplified in the range of $\pm 3.2768 \mathrm{mV}$ at a resolution of $0.1 \mu \mathrm{V}$, and filtered between 0.16 and 70 Hz. EEG data were digitally stored on hard disk for further offline analyses.

\section{Osc-tDCS}

Exp. 1 (so-tDCS) - In three separated sessions, participants were assigned to one of the following conditions: anodal, cathodal, or sham osc-tDCS. In this study, the frequency of stimulation was $0.8 \mathrm{~Hz}$. The stimulation was applied via two water-soaked sponge circular electrodes (diameter: $1.2 \mathrm{~cm}$ ) connected to a battery-operated stimulator system (BrainSTIM, EMS medical).

In the two active conditions (anodal and cathodal), a sinusoidal oscillating current with frequency of 0.8 $\mathrm{Hz}$ was applied for $10 \mathrm{~min}$ (10 sec ramp in and $10 \mathrm{~s}$ ramp out). Current intensity ranged from a minimum of $0 \mathrm{~mA}$ to a maximum of $0.6 \mathrm{~mA}$ (maximum current density: $0.531 \mathrm{~mA} / \mathrm{cm}^{2}$ ).

In the anodal condition, the anode was placed over $\mathrm{Fz}$ while the cathode, the reference electrode, was placed on the right deltoid muscle (Cogiamanian et al., 2007). The reference electrode arrangement was chosen in order to disentangle the single-polarity contribution to the cortical effects, and to describe it without confounding biases arising from two simultaneous cortical stimulations of opposite polarities involved when the reference electrode is placed on the scalp [e.g., on supraorbital region (Nasseri et al., 2013)]. In the cathodal condition, the polarity of stimulation and reference electrodes were reversed. In the sham condition, electrodes placement and current features were identical to the active conditions, but the stimulator was turned off after $10 \mathrm{sec}$. 


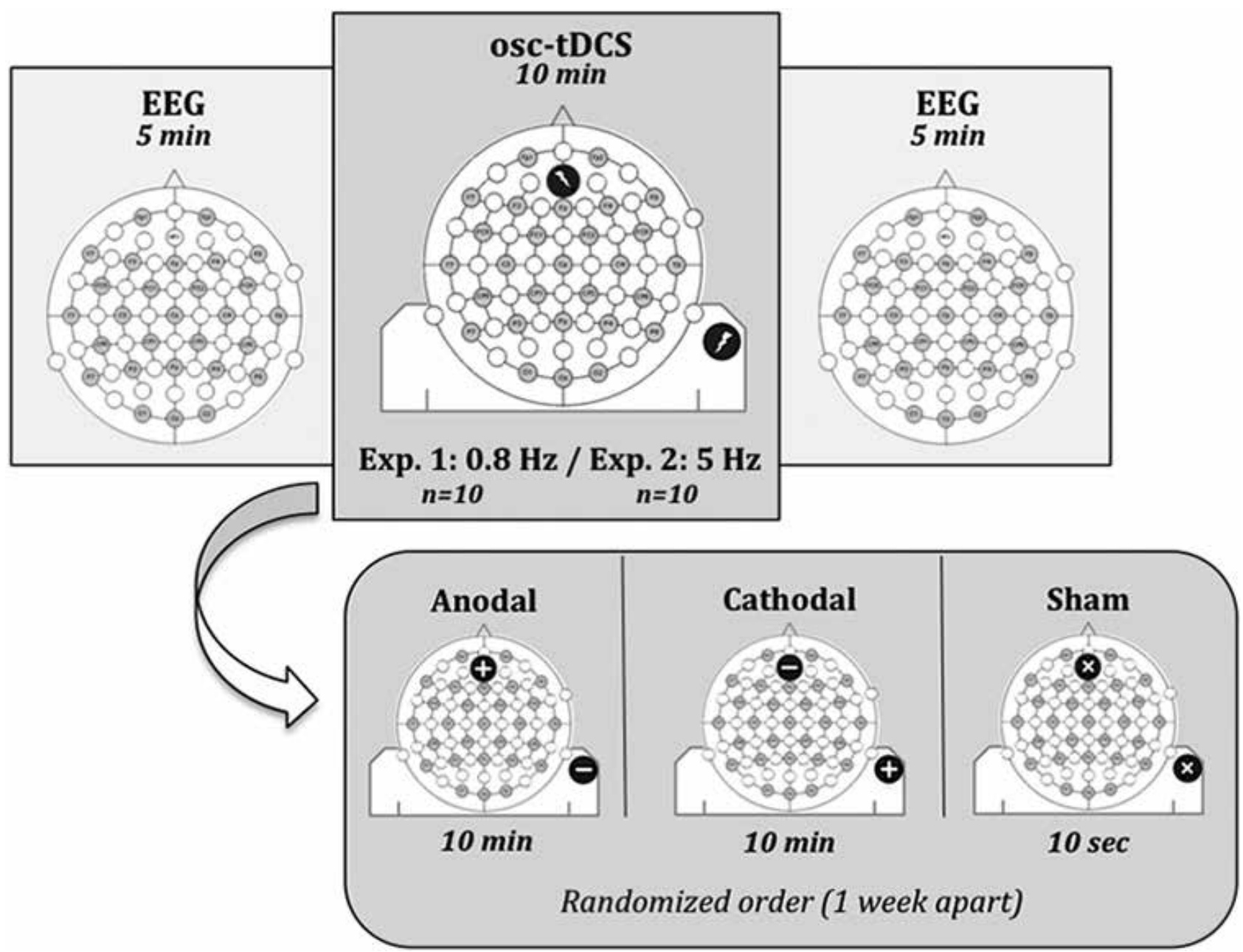

Fig. 1. - Experimental design. Upper part: Timeline of the experimental session [EEG recording montage (grey circles) and stimulation electrodes montage (black circles)]; Lower Part: Stimulation conditions within the two experiments.

Exp. 2 ( $\theta$-tDCS) - Any aspect of the procedure was identical to Exp. 1, with the only difference of the frequency of the osc-tDCS, which was set at $5 \mathrm{~Hz}$.

\section{Data analysis}

\section{Detection of oscillatory activity}

For each experimental session, after removal of 2 sec epochs with ocular and/or muscle artefacts by offline visual inspection, the BOSC analysis has been performed on the EEG signals recorded from each scalp location before and after stimulation. For a given frequency, $f$, a portion of signal is defined as rhythmic (and so detected) if the wavelet power at $f$ exceeds a power threshold, $\mathrm{P}_{\mathrm{T}}(f)$, for a time greater than a duration threshold, $\mathrm{D}_{\mathrm{T}}$ (set to 3 cycles in our analysis, $\left.\mathrm{D}_{\mathrm{T}}=3 / f\right)$. The $\mathrm{P}_{\mathrm{T}}(f)$ is derived from the estimated background spectrum that is assumed to be colored noise, Power $(f)=A f^{-\alpha}$, typical of natural autocorrelated signals (Schlesinger and West, 1998). Given this assumption, the EEG background spectrum can be fitted with a linear regression in log-log coordinates. The estimated mean at each frequency was then calculated from the regression and used as mean of the expected power values $\chi^{2}(2)$ distribution. $\mathrm{P}_{\mathrm{T}}(f)$ was set to the $95^{\text {th }}$ percentile of this theoretical probability distribution. The proportion of time in which significant oscillations at the given frequency, $f$, are detected is termed $\mathrm{P}_{\text {episode }}(f)$ (Caplan et al., 2001).

For each EEG recording and scalp electrode, one mean $\mathrm{P}_{\text {episode }}$ value was obtained at each frequency. Mean 
$\mathrm{P}_{\text {episode }}$ values corresponding to the stimulation frequencies $\left[\mathrm{P}_{\text {episode }}\left(f_{\text {Stim }}\right)\right.$ with $\left.f_{\text {Expl }}=0.81 \mathrm{~Hz} ; f_{\text {Exp } 2}=5.3 \mathrm{~Hz}\right]$ were considered as dependent variables. Individual mean $\mathrm{P}_{\text {episode }}\left(f_{\text {Stim }}\right)$ variations at the stimulation frequency was defined as the ratio between $\mathrm{P}_{\text {episode }}\left(f_{\text {Stim }}\right)$ after and before the stimulation (Post-/Pre-Stimulation).

\section{Effect of polarity}

Mean $\mathrm{P}_{\text {episode }}\left(f_{\text {Stim }}\right)$ variations obtained for each protocol within the two experiments were submitted to one-way repeated measure Analyses of Variance (ANOVAs) comparing the three conditions (Anodal, Cathodal, Sham), separately for each scalp location. To adjust the $\alpha$ value for multiple comparisons, the Bonferroni correction was applied considering the mean correlation between the dependent variables (Perneger, 1998; Sankoh et al., 1997). Given the mean correlation between the dependent variables in Exp. $1(\mathrm{r}=0.11)$ and in Exp. $2(\mathrm{r}=0.11), \alpha$ level was adjusted to 0.003 in both cases. Post-hoc tests have been carried out by paired t-tests. In case of significant effects at the omnibus ANOVA, significance of post-hoc tests was set at $\mathrm{p} \leq 0.05$.

\section{Effect of frequency}

To assess the effect of frequency, mean $\mathrm{P}_{\text {episode }}\left(f_{\text {Stim }}\right)$ variations of the two experiments were expressed as ratio between active/sham conditions. Then, these ratios were submitted to two-way mixed design ANOVAs, Frequency $(0.8 \mathrm{~Hz}$ vs. $5 \mathrm{~Hz})$ x Polarity (Anodal vs. Cathodal), with the second factor as a repeated measure. ANOVAs were carried out separately for each scalp location and for the bins corresponding to the two stimulation frequencies, i.e. 0.81 $\mathrm{Hz}$ and $5.3 \mathrm{~Hz}$. Considering the mean correlation between the dependent variables $(r=0.20), \alpha$ level was adjusted to 0.001 . Also in this case, post-hoc tests have been carried out by paired t-tests $(\mathrm{p} \leq 0.05)$.

\section{Results}

\section{Polarity of stimulation (anodal vs. cathodal vs. sham)}

\section{Experiment 1: so-tDCS}

Figure 2 shows the topography of mean $\mathrm{P}_{\text {episode }}\left(f_{\text {Expl }}=\right.$ $0.81 \mathrm{~Hz}$ ) variations in the three experimental conditions (anodal, cathodal and sham) during which so-
tDCS has been applied. The greater changes induced by the anodal stimulation involve decreases in oscillatory activity (4.8-6.9\%) across all the frontal sites. No variations are apparent after cathodal stimulation but an increase in slow oscillations at T8 (6.1\%). However, statistical comparisons do not show any significant difference at any cortical site after the Bonferroni's correction for multiple comparisons (Figure 3).

\section{Experiment 2: $\theta$-tDCS}

The topographic distribution of mean $\mathrm{P}_{\text {episode }}\left(f_{\text {Exp } 2}=\right.$ $5.3 \mathrm{~Hz}$ ) variations corresponding to the three stimulation conditions (anodal, cathodal and sham) of experiment $2(\theta$-tDCS $)$ and corresponding statistical comparisons are shown in Figures 4 and 5, respectively. Also in this case, no effect of polarity of $5 \mathrm{~Hz}$ stimulation was found on the oscillatory activity at the corresponding frequency.

\section{Frequency of stimulation ( $5 \mathrm{~Hz} v s .0 .8 \mathrm{~Hz}$ )}

According to the results of the two experiments, no significant main effect of the polarity of stimulation, while significant main effects for the frequency of stimulation have been found (Figure 6). In detail, we find a significant increase of slow oscillations $(0.81 \mathrm{~Hz})$ after $5 \mathrm{~Hz}$ compared to $0.8 \mathrm{~Hz}$ stimulation over Cp2 $\left(\mathrm{F}_{1,18}=19.97, \mathrm{p}=0.0004\right)$ and an increase of theta oscillations after $5 \mathrm{~Hz}$ compared to $0.8 \mathrm{~Hz}$ stimulation over $\mathrm{Fc} 1\left(\mathrm{~F}_{1,18}=26.93, \mathrm{p}=0.0001\right)$. Although no Frequency x Polarity interaction was significant after the Bonferroni correction, a close to significance effect was detected for the slow oscillations at the Fz stimulation site $\left(\mathrm{F}_{2,18}=11.02\right.$, $\mathrm{p}=0.004$ ), explained by greater slow oscillations after the $5 \mathrm{~Hz}$ than $0.8 \mathrm{~Hz}$ anodal stimulation $\left(\mathrm{t}_{18}=\right.$ $2.74, \mathrm{p}=0.01$ ).

\section{Discussion}

The current study assessed the effects on rhythmic cortical activity of oscillatory tDCS during wakefulness at rest comparing different stimulation frequencies $(0.8 \mathrm{~Hz}$ and $5 \mathrm{~Hz}$ ) and polarities (anodal and cathodal). Assuming that osc-tDCS acts on cortical activity by inducing resonance effects in the stimulated network (Ozen et al., 2010; Fröhlic and McCormick, 2010) and that its effectiveness is affected by the consistence between stimulation 
frequency and brain-state, we evaluated the frequency-specific effect of $\theta$-tDCS and so-tDCS on "genuine" oscillatory activity at the stimulation frequency. Considering EEG oscillations as the dependent variable, we found a significant local frequency-dependent increase of theta rhythmic activity $(5.3 \mathrm{~Hz})$ after $\theta$-tDCS compared to so-tDCS. Moreover, $\theta$-tDCS more than so-tDCS was able to induce increases of oscillatory activity not only at the stimulation frequency but also in the slow oscillations' range $(0.81 \mathrm{~Hz})$, suggesting a more general synchronizing effect on cortical activity.

\section{Highlighting the frequency-specific effect} of osc-tDCS on cortical oscillations

The effects of oscillatory tDCS involve both frequency-specific and cross-frequency modulation of brain activity (Marshall et al., 2006; 2011; Kirov et al., 2009; Nueling et al., 2012; Pahor and Jaušovec, 2014). Frequency-specific increases in slow oscillations $(0.5-1 \mathrm{~Hz})$ have been reported after osc-tDCS at $0.75 \mathrm{~Hz}$ applied on frontal areas during NREM sleep, together with cross-frequency increases in alpha rhythm (8-12 Hz) (Marshall et al., 2006). The same stimulation during wakefulness confirmed the presence of frequency-specific effects, but also induced a cross-frequency enhancement in theta band (4-8 Hz) (Kirov et al., 2009). Similarly, a stimulation at $10 \mathrm{~Hz}$ on temporal areas resulted in increases in alpha activity, with a maximum at the stimulation frequency, associated with increases in delta band (1-4 Hz) (Neuling et al., 2012).

Here, we show a frequency-specific effect of the $5-\mathrm{Hz}$ stimulation compared to the $0.8 \mathrm{~Hz}$ by measuring the "genuine" EEG oscillatory activity. In particular, the effect was localized perifocal to the stimulation site; therefore, it could be reasonably interpreted as an after-effect of resonance phenomena induced by the stimulation on the target area. Conventional FFT analysis of the same EEG recordings (these data will be published elsewhere) failed to reveal this frequency-specific effect of $\theta$-tDCS, confirming the informative contribution that can arise from a specific investigation on cortical oscillations.

It is worth nothing, anyway, that the extent of the induced changes of spontaneous oscillatory activity are actually small, probably because of the scarcity of oscillatory activity in theta range during wakeful- ness, when cortical oscillations are mostly expressed within the alpha frequency band.

We also found a cross-frequency effect of $5-\mathrm{Hz}$ stimulation on slow oscillations $(0.81 \mathrm{~Hz})$. Although this result may not be explained by resonance effects, it nevertheless indicates that 5-Hz compared to $0.8-\mathrm{Hz}$ stimulation specifically enhances EEG synchronization.

\section{The effect of polarity}

It has been shown that tDCS polarity, i.e. the direction of the applied electric field, determines the direction of changes induced on membrane potential at neuronal level (Bindman et al.,1964; Purpura and Mcmurtry, 1965; Bikson et al., 2004; Fröhlic and McCormick, 2010). In particular, the stimulation induces somatic polarization in neural populations underlying the electrode with anodal polarity, resulting in increases of firing rates, while opposite effects are induced under the electrode with cathodal polarity. According to this view, oscillatory tDCS should result in a periodic shift of neuronal membrane potentials (Bergmann et al., 2009) driving neurons to oscillate at the stimulation frequency whether the stimulation polarity is anodic or cathodic. Nevertheless, the possible finding of different effects depending on the stimulation polarity would indicate that the resonance effects associated with the oscillating component of the stimulation and the changes in cortical excitability associated with the direct component of the stimulation interact in determining the overall stimulation effect.

In the present study, the extra-cephalic placement of the reference electrode allows to disentangle the single polarity contribution to cortical effects of the stimulation overpassing the confounding biases arising from two simultaneous stimulations of opposite polarity associated with the cephalic reference commonly adopted in most of the previous studies (Marshall et al., 2006; 2011; Kirov et al., 2009; Neuling et al., 2012). Specifically, the de-/ hyper-polarization of areas close to the reference electrode, in a totally cephalic stimulation montage, likely affects the outcomes of the main stimulation preventing from the possibility to evaluate the actual role of the polarity in shaping the cortical effects.

However, the current study failed to show any clear polarity-dependency of the effects. This lack of polarity effects resembles the results of a recent 


\section{$0.8 \mathrm{~Hz}$}
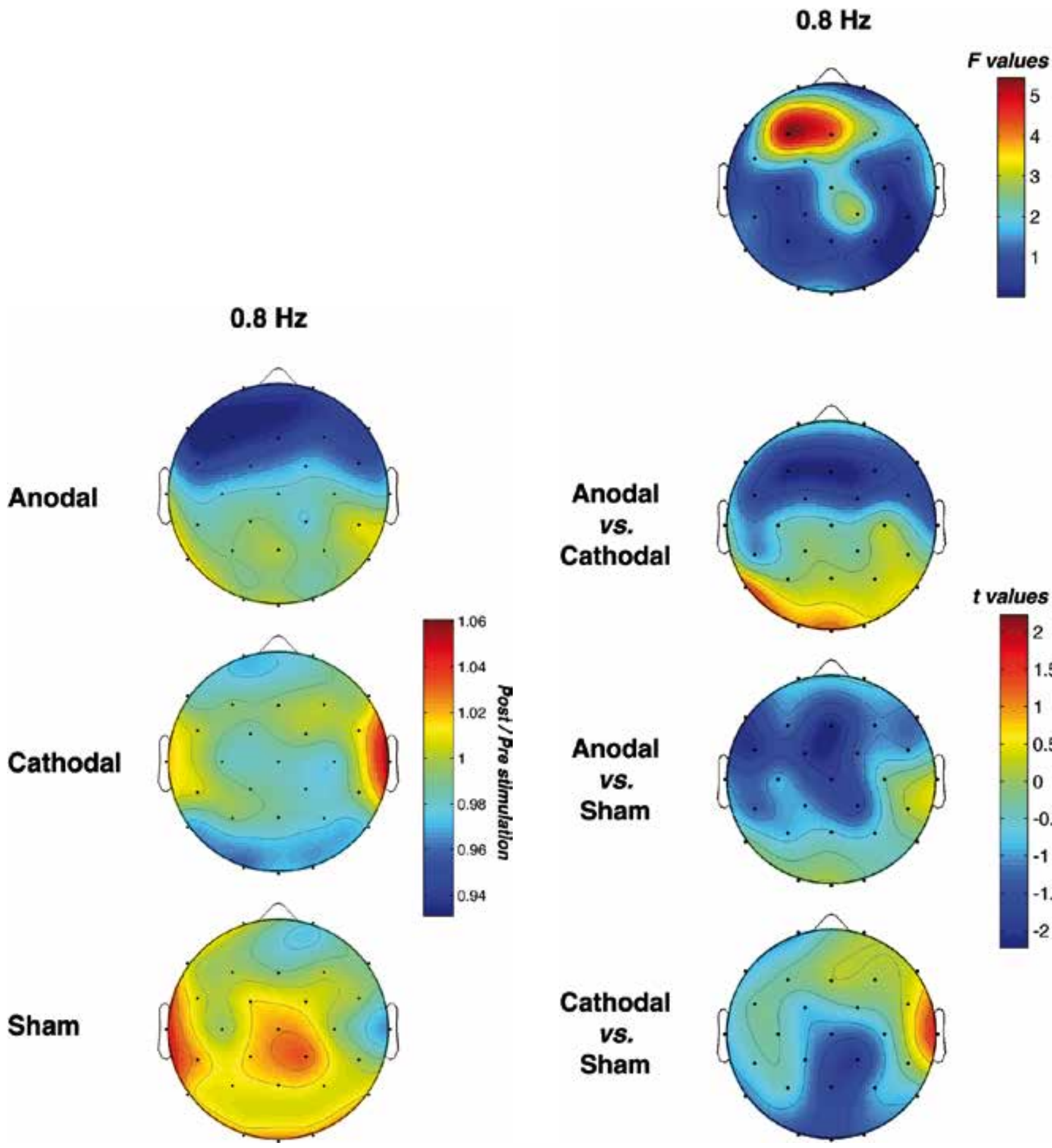

Fig. 2. - Topographic distribution of mean relative changes in oscillatory activity after anodal, cathodal and sham so-tDCS, expressed as ratio between post- and pre-stimulation $\mathrm{P}_{\text {episode }}\left(f_{\text {stim }}\right)$, i.e. proportion of time in which significant oscillations at the stimulation frequency are detected $\left(f_{\text {stim }}=0.81 \mathrm{~Hz}\right)$. Average values are color coded and plotted at the corresponding position on the planar projection of the scalp surface and are interpolated (biharmonic spline) between electrodes. Values $>1$ represent increase in oscillatory activity after stimulation relative to before stimulation and vice versa for values $<1$. The maps are based on the 28 unipolar EEG derivations of the international 10-20 system with linked mastoid reference.

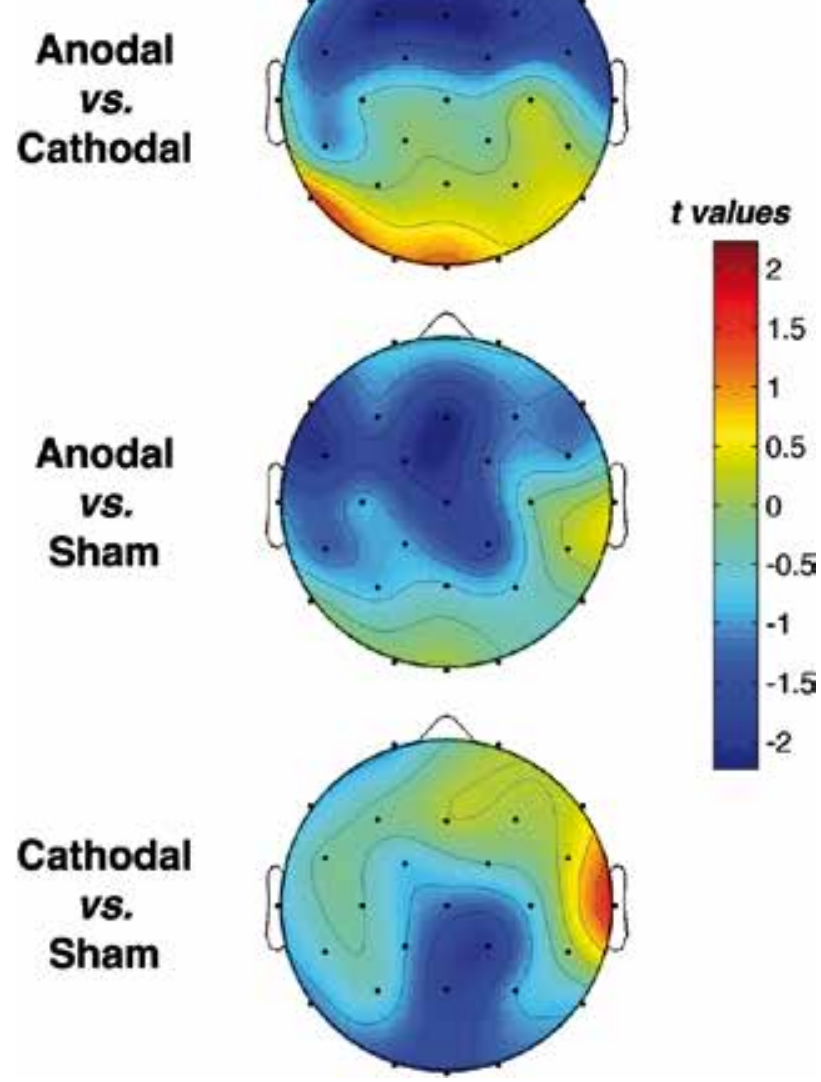

Fig. 3. - Statistical maps of comparisons between anodal, cathodal and sham so-tDCS. Values are color coded and plotted at the corresponding position on the planar projection of the scalp surface and are interpolated (biharmonic spline) between electrodes. Upper part: Results of one-way omnibus ANOVAs comparing the three conditions.

Lower part: Results of post-hoc comparisons (paired t-tests). Positive coefficients indicate a greater increase of oscillatory activity in the anodal compared to cathodal, in the anodal compared to sham, and in the cathodal compared to sham stimulation, respectively. 


\section{$5.3 \mathrm{~Hz}$}

Anodal
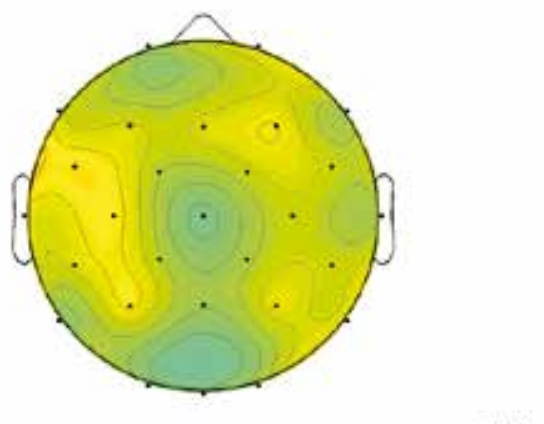

\section{Cathodal}

\section{Sham}

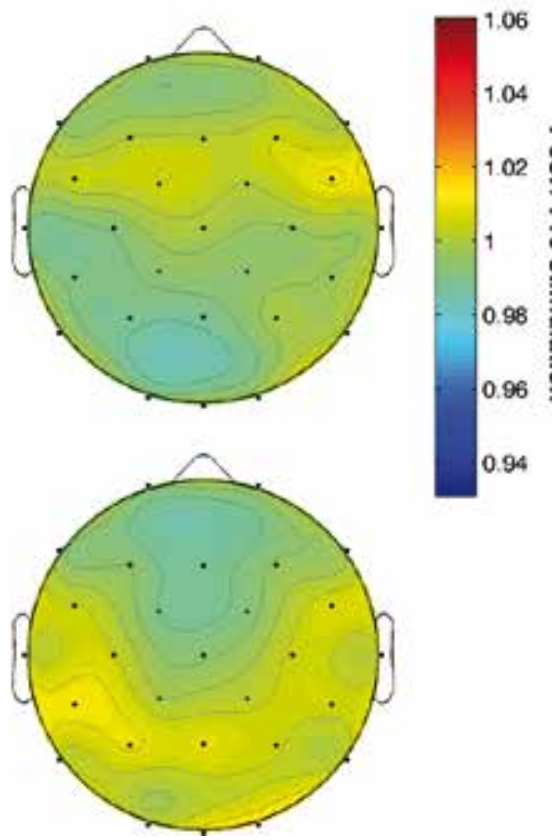

Fig. 4. - Topographic distribution of mean relative changes in oscillatory activity after anodal, cathodal and sham $\theta$-tDCS, expressed as ratio between post- and pre-stimulation $\mathrm{P}_{\text {episode }}\left(f_{\text {stim }}\right)$, i.e. proportion of time in which significant oscillations at the stimulation frequency are detected $\left(f_{\text {stim }}\right.$ $=5.3 \mathrm{~Hz}$ ). Average values are color coded and plotted at the corresponding position on the planar projection of the scalp surface and are interpolated (biharmonic spline) between electrodes. Values $>1$ represent increase in oscillatory activity after stimulation relative to before stimulation and vice versa for values $<1$. The maps are based on the 28 unipolar EEG derivations of the international 10-20 system with linked mastoid reference.
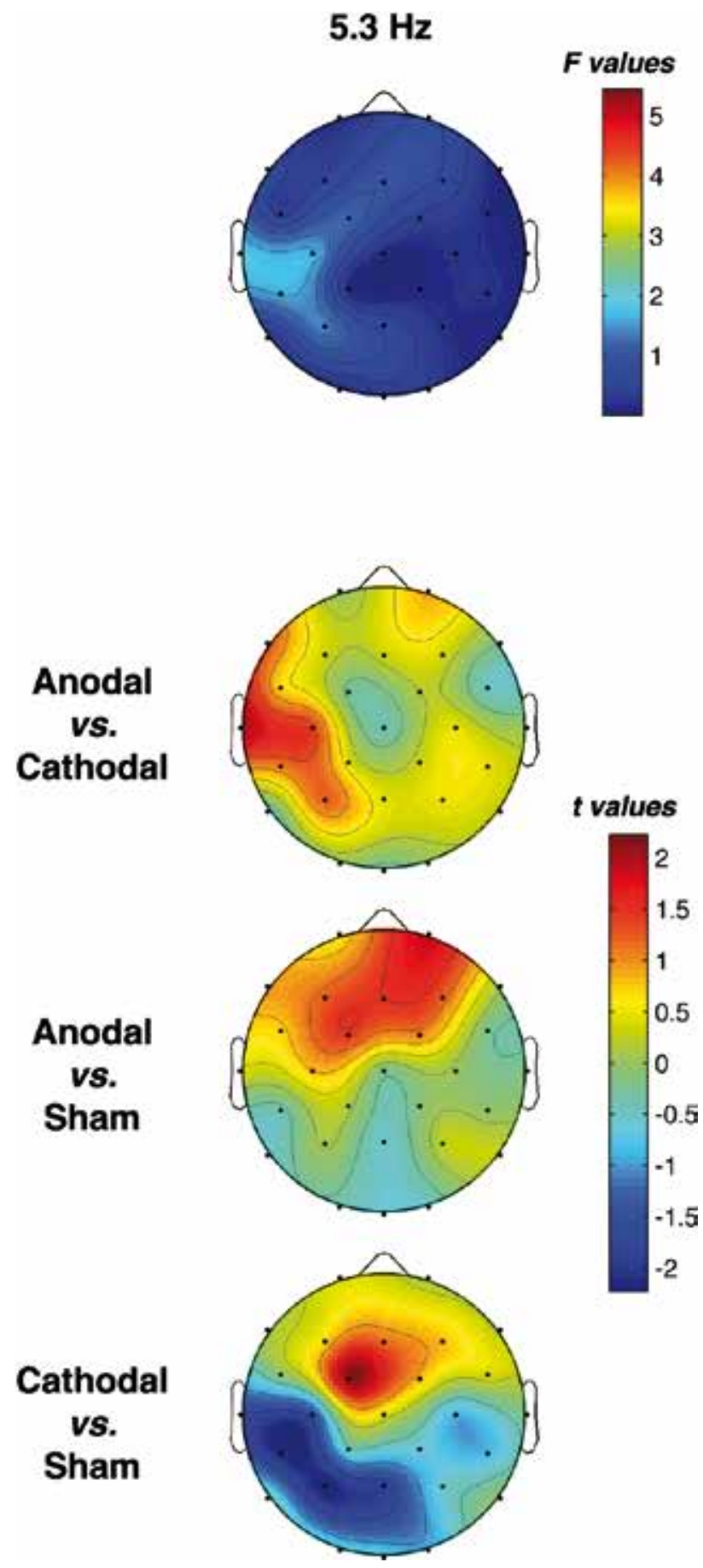

Fig. 5. - Statistical maps of comparisons between anodal, cathodal and sham stimulation ( $\theta-\mathrm{tDCS}$ ) conditions. Values are color coded and plotted at the corresponding position on the planar projection of the scalp surface and are interpolated (biharmonic spline) between electrodes.

Upper part: Results of one-way omnibus ANOVAs comparing the three conditions.

Lower part: Results of post-hoc comparisons (paired t-tests). Positive coefficients indicate a greater increase of oscillatory activity in the anodal compared to cathodal, in the anodal compared to sham, and in the cathodal compared to sham stimulation, respectively. 
$0.8 \mathrm{~Hz}$
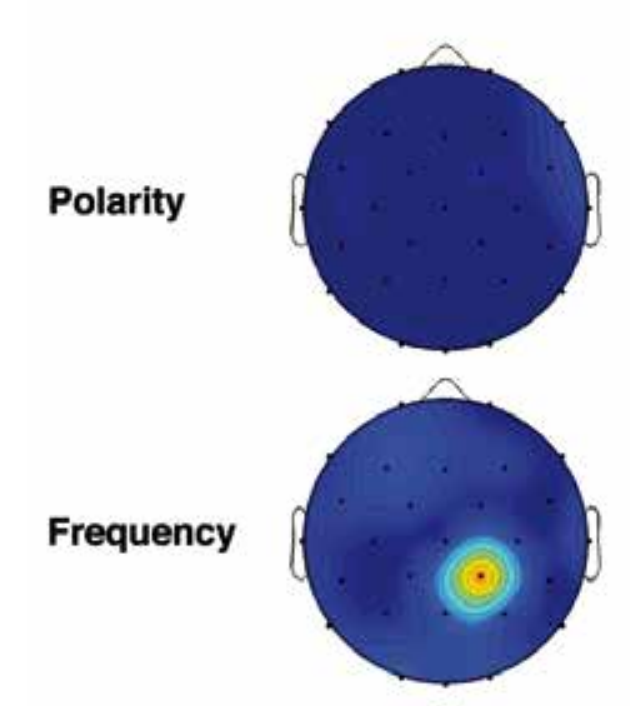

Polarity

$\mathbf{x}$

Frequency
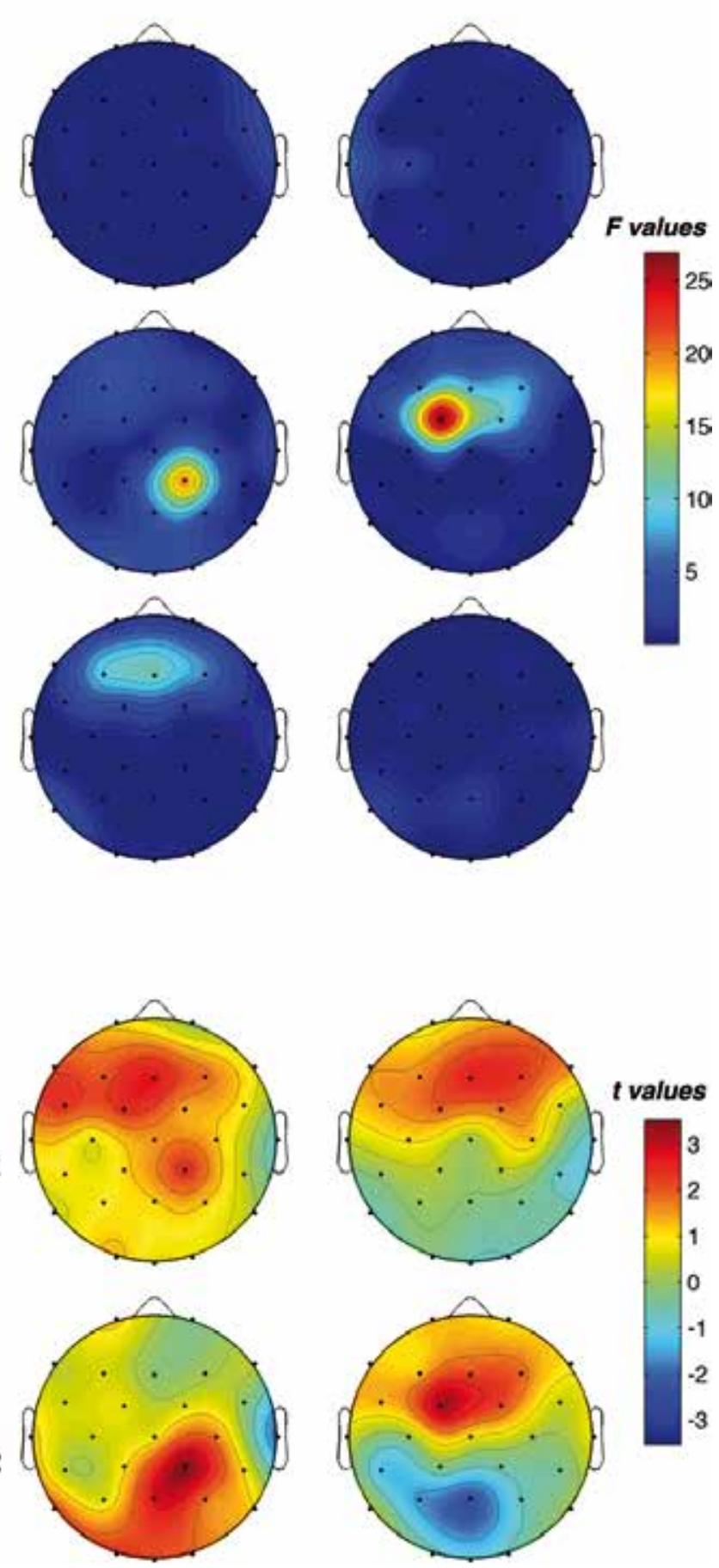

Fig. 6. - Statistical maps of the comparisons between 0.8 vs. $5 \mathrm{~Hz}$ osc-tDCS conditions. Planned comparisons have been carried our separately for anodal and cathodal stimulations. Values are color coded and plotted at the corresponding position on the planar projection of the scalp surface and are interpolated (biharmonic spline) between electrodes. Maps are plotted for the following frequency bin correspondent to the two stimulation frequencies $(0.81 \mathrm{~Hz}, 5.3 \mathrm{~Hz})$.

Upper part: Results of two-way mixed design ANOVAs, Frequency $(0.8 \mathrm{~Hz}$ vs. $5 \mathrm{~Hz}$ ) x Polarity (Anodal vs. Cathodal), with the second factor as a repeated measure. The main effects are reported in the first two rows, and the interactions are depicted in the third row.

Lower part: Results of the planned comparisons (paired t-tests) between $0.8 \mathrm{vs.} 5 \mathrm{~Hz}$ frequency of osc-tDCS for anodal and cathodal conditions, respectively. Positive coefficients indicate a greater increase of oscillatory activity after a $5-\mathrm{Hz}$ compared to $0.8 \mathrm{~Hz}$ stimulation. 
study using large-scale simulations of cortical networks, which reported that both polarizing-only and hyperpolarizing-only oscillating stimulations might modulate network oscillations by means of slightly different mechanisms (Ali et al., 2013). They also found that a stimulation with alternating current could be even more effective in entraining network oscillations (Ali et al., 2013), suggesting that transcranial alternating current stimulation (tACS) protocols could result in greater outcomes, at least when the aim of the study is the enhancement of cortical activity at a given frequency.

\section{Conclusions}

In conclusion, we find larger frequency-specific and cross-frequency effects of $5-\mathrm{Hz}$ compared to $0.8-\mathrm{Hz}$ stimulation, consistent with a more effective induction of EEG synchronization during wakefulness by means of a stimulation in theta range.

Although characterized by a relatively small magnitude, our finding remarks the importance of detecting also "genuine" oscillatory activity, since the BOSC method highlighted some effects not shown by conventional FFT analyses. For this reason, we suggest in future studies involving oscillating stimulations to combine measurement of EEG power and EEG oscillations.

\section{Acknowledgement}

This work was supported by a grant to LDG from "Ministero della Salute" (Ministry of Health) RF-2009-1528677, and by a grant to LDG from "Progetti di Ricerca di Ateneo 2012 (C26A1254AT), 2013 (C26A139JL4), 2014 (C26A143ZEP)" (University of Rome Sapienza).

\section{References}

Ali M.M., Sellers K.K., Frohlich F. Transcranial alternating current stimulation modulates largescale cortical network activity by network resonance. J. Neurosci., 33: 11262-11275, 2013.

Bergmann T.O., Groppa S., Seeger M., Mölle M., Marshall L., Siebner H.R. Acute changes in motor cortical excitability during slow oscillatory and constant anodal transcranial direct current stimulation. J. Neurophysiol., 102: 2303-2311, 2009.

Bikson M., Inoue M., Akiyama H., Deans J.K., Fox J.E., Miyakawa H., Jefferys J.G. Effects of uniform extracellular DC electric fields on excitability in rat hippocampal slices in vitro. J. Physiol., 557: 175-190, 2004.

Bindman L.J., Lippold O.C., Redfearn J.W. The action of brief polarizing currents on the cerebral cortex of the rat (1) during current flow and (2) in the production of long-lasting after-effects. $J$. Physiol., 172: 369-382, 1964.

Cogiamanian F., Marceglia S., Ardolino G., Barbieri S., Priori A. Improved isometric force endurance after transcranial direct current stimulation over the human motor cortical areas. Eur. J. Neurosci., 26: 242-249, 2007.

Caplan J.B., Kahana M.J., Raghavachari S., Madsen J.R. Distinct patterns of brain oscillations underlie two basic parameters of human maze learning. $J$. Neurophysiol., 86: 368-380, 2001.

Caplan J.B. and Glaholt M.G. The roles of EEG oscillations in learning relational information. Neuroimage, 38: 604-616, 2007.

Caplan J.B., Bottomley M., Kang P., Dixon R.A. Distinguishing rhythmic from non-rhythmic brain activity during rest in healthy neurocognitive aging. NeuroImage, 112: 341-352, 2015.

Fröhlich F. and McCormick D.A. Endogenous electric fields may guide neocortical network activity. Neuron, 67: 129-143, 2010.

Gutfreund Y., Yarom Y., Segev I. Subthreshold oscillations and resonant frequency in guinea-pig cortical neurons: physiology and modelling. $J$. of Physiol., 483: 621-640, 1995.

Hughes A.M., Whitten T.A., Caplan J.B., Dickson C.T. BOSC: a Better OSCillation detection method, extracts both sustained and transient rhythms from rat hippocampal recordings. Hippocampus, 22: 1417-1428, 2012.

Hutcheon B. and Yarom Y. Resonance, oscillation and the intrinsic frequency preferences of neurons. Trends Neurosci., 23: 216-222, 2000.

Kirov R., Weiss C., Siebner H.R., Born J., Marshall L. Slow oscillation electrical brain stimulation during waking promotes EEG theta activity and memory encoding. Proc. Natl. Acad. Sci. U.S.A., 106: 15460-15465, 2009.

Marshall L., Helgadóttir H., Mölle M., Born J. Boosting slow oscillations during sleep potentiates memory. Nature, 444: 610-613, 2006. 
Marshall L., Kirov R., Brade J., Mölle M., Born J. Transcranial electrical currents to probe EEG brain rhythms and memory consolidation during sleep in humans. PLoS ONE, 6: e16905, 2011.

Marzano C., Ferrara M., Mauro F., Moroni F., Gorgoni M., Tempesta D., Cipolli C., De Gennaro L. Recalling and forgetting dreams: Theta and alpha oscillations during sleep predict subsequent dream recall. J. Neurosci., 3: 6674-6683, 2011.

Marzano C., Moroni F., Gorgoni M., Nobili L., Ferrara M., De Gennaro L. How we fall asleep: regional and temporal differences in electroencephalographic synchronization at sleep onset. Sleep Med., 14: 1112-1122, 2013.

Moroni F., Nobili L., De Carli F., Massimini M., Francione S., Marzano C., Proserpio P., Cipolli C., De Gennaro L., Ferrara M. Slow EEG rhythms and inter-hemispheric synchronization across sleep and wakefulness in the human hippocampus. Neuroimage, 60: 497-504, 2012.

Nasseri P., Nitsche M.A., Ekhtiari H. A framework for categorizing electrode montages in transcranial direct current stimulation. Front. Hum. Neurosci., 9: 54, 2015.

Neuling T., Rach S., Wagner S., Wolters C.H., Herrmann C.S. Good vibrations: oscillatory phase shapes perception. Neuroimage, 63: 771-778, 2012.

Ozen S., Sirota A., Belluscio M.A., Anastassiou C.A., Stark E., Koch C., Buzsáki G. Transcranial electric stimulation entrains cortical neuronal populations in rats. J. Neurosci., 30: 11476-11485, 2010.

Perneger T.V. What's wrong with Bonferroni adjustments. B.M.J., 316: 1236-1238, 1998.

Purpura D.P. and Mcmurtry J.G. Intracellular activities and evoked potential changes during polarization of motor cortex. J. Neurophysiol. 28: 166-185, 1965.

Radman T., Su Y., An J.H., Parra L.C., Bikson M. Spike timing amplifies the effect of electric fields on neurons: implications for endogenous field effects. J. Neurosci., 27: 3030-3036, 2007.

Reato D., Rahman A., Bikson M., Parra L.C. Low-intensity electrical stimulation affects network dynamics by modulating population rate and spike timing. J. Neurosci., 30: 15067-15079, 2010.

Sankoh A.J., Huque M.F. Dubey S.D. Some comments on frequently used multiple endpoint adjustments methods in clinical trials. Stat. Med., 16: 2529-2542, 1997.

Schlesinger M.F. and West B.J.. 1/f versus 1/f a noise. pp. 320-324. In: Stanley E.H., Ostrowsky E.H. (Eds.) Random Fluctuations and Pattern Growth: Experiments and Models. Kluwer, Dordrecht, 1988.

Whitten T.A., Hughes A.M., Dickson C.T., Caplan J.B. A better oscillation detection method robustly extracts EEG rhythms across brain state changes: the human alpha rhythm as a test case. NeuroImage, 54: 860-874, 2011. 